\title{
MIXING AND COMBUSTION IN SUPERSONIC NEAR-WALL SHEAR FLOWS
}

\author{
ROMAN S. SOLOMATIN ${ }^{1}$, ILYA V. SEMENOV ${ }^{2}$ AND IGOR S. MENSHOV \\ ${ }^{1}$ FSC Scientific Research Institute for System Analysis RAS, \\ 117218, Moscow, Nakhimovskiy avenue, 36/1, solomatin@ niisi.ras.ru \\ ${ }^{2}$ FSC Scientific Research Institute for System Analysis RAS, \\ 117218, Moscow, Nakhimovskiy avenue, 36/1, ivsemenov@niisi.ras.ru \\ ${ }^{3}$ Keldysh Institute for Applied Mathematics RAS, \\ 125047, Moscow, Miusskaya sq., 4, menshov@kiam.ru
}

Key words: supersonic shear flow, turbulent mixing, combustion

Summary: Mixing, ignition, and flame stabilization in a shear supersonic hydrogen-air flow is numerically studied with the RANS model of Spalart and Allmaras. A detailed chemical kinetics of hydrogen-air combustion is used in the calculations. The system of governing equations is solved with a hybrid explicit-implicit time marching scheme and the LU-SGS method. Modelling of hydrogen injection into the $M=2.44$ vitiated air flow in the model Burrows-Kurkov combustor is carried out for $2 D$ and $3 D$ cases. The numerical results obtained are compared with available experimental data.

\section{INTRODUCTION}

The development of high-effective jet engines with high thrust and low losses is becoming an important and actual problem in recent times. An approach to increase the effectiveness of engines is to increase the fuel-oxidizer mixture combustion rate. Typical velocities in modern combustion chambers reach supersonic values for high-speed propulsion vehicles. At these regimes, combustion of non-premixed gas flows meets some problems - high velocities and short timings lead to incomplete mixing and the formation of gradients in the species concentrations distributions which results in inhomogeneous mixture; the flame zone configuration is specified by the mixing and ignition processes. Furthermore, supersonic viscous boundary layers forming on the walls have considerable thickness. The flow velocity decrease in the near wall region may give a critical impact on the process of combustion.

In the present work, we analyze the process of the near wall supersonic gas combustion in the Burrows and Kurkov model combustion chamber. Some experimental studies of this combustor were published in 1971 [1] and 1973 [2]. These experimental data have been so far used for validation mathematical models and numerical methods [3-6]. Application of different turbulence models, kinetics schemes and TCI (turbulence-chemistry interaction) models were investigated in $[7,8]$. Nowadays, due to development of computational 
resources, it's becoming possible to carry out numerical simulations based on more advanced DES (detached eddy simulation) and DDES approaches [9, 10].

The present paper is devoted to numerical modelling of physical-chemical processes in turbulent spatial wall-confined flows at supersonic velocities. A mixing model based on the RANS approach and the Spalart-Allmaras (SA) turbulent model is used. The validation of the model is presented in [11]. The mixing model is coupled with a detailed kinetics mechanism for hydrogen-air combustion and applied to ignition and combustion modelling in the Burrows-Kurkov combustion chamber.

\section{MATHEMATICAL MODEL}

The governing equations are the modified Reynolds averaged Navier-Stokes equations for multicomponent chemically reactive gas mixture that include balance equations for species mass, total momentum, and total energy. This system of equations is closed with the Spalart-Allmaras (SA) turbulence model equation, which represents the transfer equation for modified turbulence viscosity [12],

$$
\frac{D \tilde{v}}{D t}=P^{v}-D^{v}+\frac{1}{\sigma}\left\{\nabla \cdot[(v+\tilde{v}) \nabla \tilde{v}]+c_{b 2}[(\nabla \tilde{v}) \cdot(\nabla \tilde{v})]\right\}
$$

Since the SA model is linear model, the Boussinesque assumption can be implemented for the components of the turbulent viscous stress tensor. The turbulent heat flux vector is defined via the Fourier law,
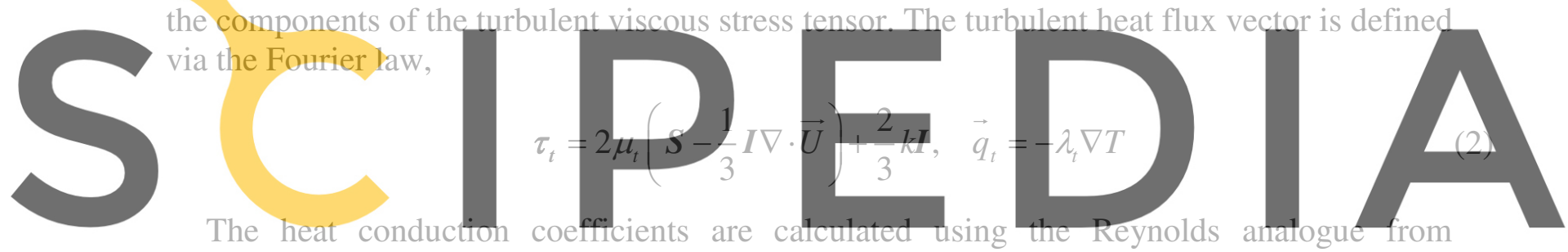

The heat conduction coefficients are calculated using the Reynolds antalogut from
propriate viscosity coefficients, Prandtl numbers, and specific heat coefficients. Diffusion is

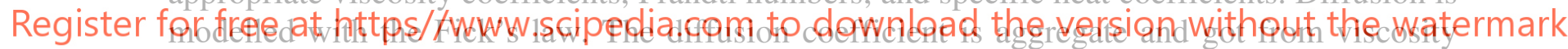
coefficients and Schmidt numbers.

Mass production rates due to chemical reactions in the species mass balance equations, $\omega_{i}$, are calculated as follows:

$$
\dot{\omega}_{i}=m_{i} \sum_{r=1}^{N_{r}}\left(b_{i, r}-f_{i, r}\right)\left[K_{r}^{f} \prod_{i}\left(\frac{\rho_{i}}{m_{i}}\right)^{f_{i, r}}-K_{r}^{b} \prod_{i}\left(\frac{\rho_{i}}{m_{i}}\right)^{b_{i, r}}\right]
$$

where $N_{r}$ is the number of chemical reactions, $f_{i, r}$ and $b_{i, r}$ are the stoichiometric coefficients for specie $i$ in the forward and backward r-th reaction, respectively, and $K_{r}^{f}, K_{r}^{b}$ are the forward and backward $r$-th reaction rate coefficients. The reaction rate coefficients are given in the Arrhenius form and depend on the kinetics mechanism. In present study detailed hydrogen-air kinetics mechanism, developed by A. Keromnes [13], is used. Current mechanism consists of 22 reversible reactions between 9 components. Three reactions from the kinetics set are pressure-dependent. Dependence is implemented in Troe form [14]. Detailed kinetics mechanism, having dependence from temperature and pressure, provides 
correct prediction of self-ignition delays in wide range of pressure, temperature and equivalence ratios.

\section{NUMERICAL METHOD}

The finite volume method is applied to solve the system of governing equations. The computational domain is divided into control volumes in the form of an arbitrary polyhedral. Each face of the control volume is characterized by its area and unit outward normal.

Time integration is performed with the hybrid explicit-implicit scheme [15] that in the explicit mode reduces to the second-order predictor-corrector scheme. Hybridization coefficient $\xi_{i}$ based on the stability criterion is calculated in each cell independently. The discrete equations are written in the following form:

$$
\begin{aligned}
& \vec{q}_{i}^{*}=\vec{q}_{i}^{n+\xi_{i}}-\frac{\Delta t}{2 V_{i}} \xi_{i} \sum_{\theta}\left(\vec{f}_{k, \theta}^{n+\xi_{i}}-\vec{g}_{k, \theta}^{n+\xi_{i}}\right) n_{\theta} s_{\theta}+\omega_{i} \Delta t \vec{S}_{i}^{n+\xi_{i}} \\
& \vec{q}_{i}^{n+1}=\vec{q}_{i}^{n}-\frac{\Delta t}{V_{i}} \sum_{\theta}\left(\vec{f}_{k, \theta}^{*}-\vec{g}_{k, \theta}^{*}\right) n_{\theta} S_{\theta}+\Delta t \vec{S}_{i}^{*}
\end{aligned}
$$

where $\vec{q}$ is the vector of conservative variables, $\vec{f}$ and $\vec{g}$ are convective and viscous flux vectors, respectively, $\vec{S}$ is the source vector. The subscript $\theta$ shows that the value is taken at the barycenter of the face $\theta, s_{\theta}$ is the area of the face $\theta$ and $\boldsymbol{n}_{\theta}$ is the unit nornual to the face $\theta . V_{i}$ is the cell volum time level.

The dual-time step schem process with parameter $\theta, s$ is the area of
Superscript $n+\xi_{i}$ m
cheme is applied to
called "pseudotime"

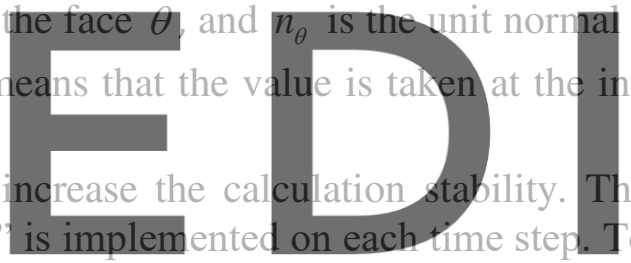
al to the face
intermediate
The itefation
To sdve we seek the steady solution to the equation:

Register for free at https//www.scipedia.comito download the version without the watermark $(1-\xi) \frac{\partial q}{\partial \tau}=-\left(\vec{q}^{n+1}-\vec{q}^{n}\right)-R^{n+1}$,

where $R^{n+1}$ is the residual,

$$
R_{i}^{n+1}=\frac{\Delta t}{V_{i}} \sum_{\theta}\left(\vec{f}_{k, \theta}^{*}-\vec{g}_{k, \theta}^{*}\right) n_{\theta} s_{\theta}-\Delta t \vec{S}_{i}^{*}
$$

Equation (5) is solved numerically with the implicit backward Euler scheme and Newton's iterations. The computational algorithm is the parallel realization of the LU-SGS method [16] that requires two (forward and backward) explicit sweeps. Authors use the method modification that doesn't require any matrix assembly and contributes memory resources economically.

The chemical kinetics equation system is solved with the Gear's method. Applied realization is DLSODE integrator [17] that is able to select integration step automatically. The obtained solution of the kinetics equations is used to evaluate source term $\vec{S}$ for the governing equations. 
Convective fluxes are approximated with the Godunov method by solving the Riemann problem on cell faces. The viscous fluxes are computed with the generalized central difference scheme.

\section{PROBLEM STATEMENT AND NUMERICAL RESULTS}

Turbulent mixing, ignition and flame stabilization in supersonic gas co-flows problem is investigated. The hydrogen flow with the velocity corresponding a Mach number $M=1$ is injected along the supersonic gas flow $M=2.44$ in the combustor. The gas is air with hydrogen combustion products (vitiated air). The combustor geometry is taken from [1] and is presented in Fig. 1. Chamber length is 14 inches $(35.6 \mathrm{~cm})$, height expands from $8.9 \mathrm{~cm}$ at the beginning to the $10.5 \mathrm{~cm}$ at the exit. The height of the hydrogen nozzle is $0.4 \mathrm{~cm}$. The problem is considered in the two- and three-dimensional statements. In 3D combustion chamber, the width is $5 \mathrm{~cm}$. Also computational domain contains a part of the isolator with a length of $17.24 \mathrm{~cm}$ and a part of the nozzle with a length of $1.5 \mathrm{~cm}$.

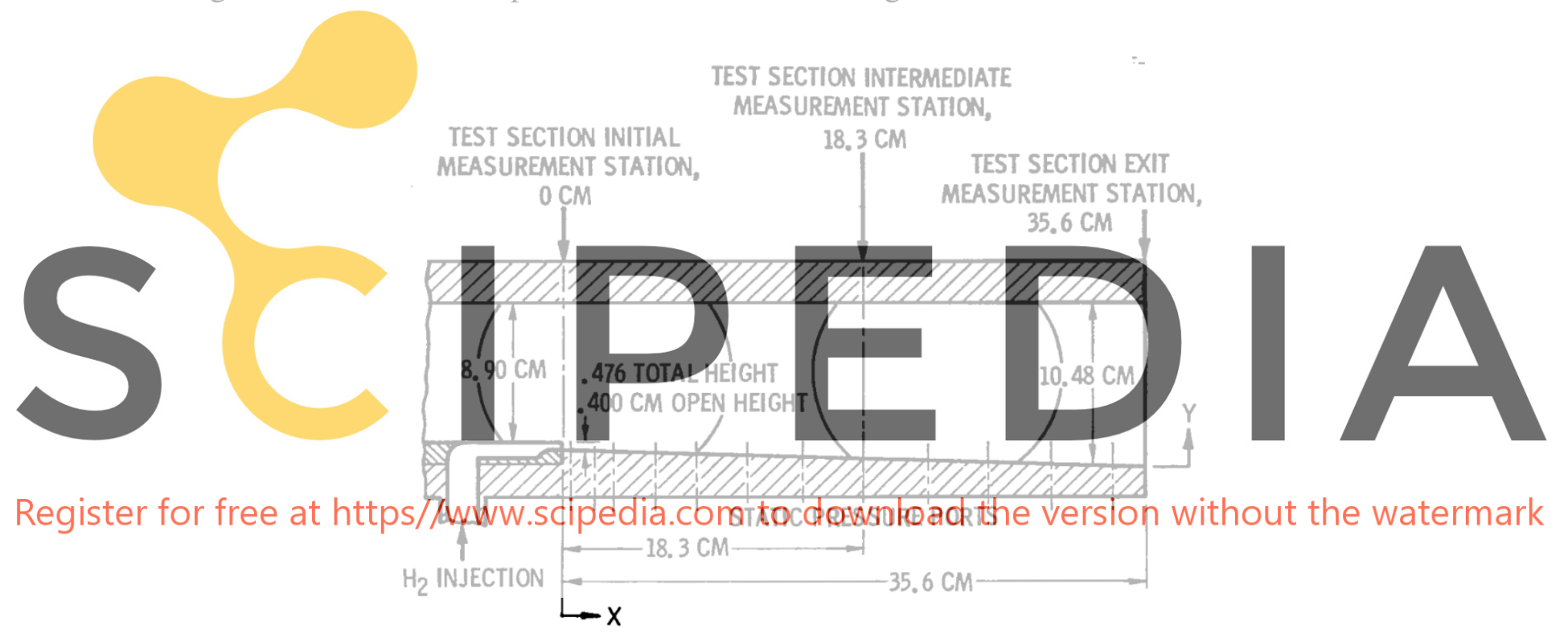

Figure 1: Combustor geometry (taken from [1])

The gas composition in terms of mass fractions [10]: $\mathrm{H}_{2}-0.0005, \mathrm{O}_{2}-0.0509, \mathrm{H}_{2} \mathrm{O}-$ $0.2162, \mathrm{~N}_{2}-0.7324$. Pitot pressure of the flow: $7.4 \mathrm{kPa}$, total temperature $-2207 \mathrm{~K}$. Gas dynamic parameters of hydrogen: $\mathrm{P}=216.675 \mathrm{kPa}$ and $\mathrm{T}=314 \mathrm{~K}$, respectively.

Mass fractions of the vitiated air components: $\mathrm{O}_{2}-0.258, \mathrm{H}_{2} \mathrm{O}-0.256, \mathrm{~N}_{2}-0.486$, which also corresponds [10]. The flow Pitot pressure is $700 \mathrm{kPa}$, the total temperature is $2160 \mathrm{~K}$. The hydrogen flow has a total pressure of $181 \mathrm{kPa}$ and a total temperature of $302 \mathrm{~K}$.

Combustor walls are supposed to be isothermal with a temperature of $298 \mathrm{~K}$ and are able to warm up to a depth of $0.017 \mathrm{~m}$. Such a depth is taken from the experimental data of heat penetration into the combustor copper walls during $2.5 \mathrm{~s}$ long experiment. To specify the inflow boundary condition for supersonic gas flow, numerical modeling of supersonic gas flow through the rectangular channel was carried out. The length of the channel was $1 \mathrm{~m}$. 
Flow parameters correspond to the parameters of the basic problem. Transversal dimensions repeat the size of chamber isolator. An auxiliary problem was also solved in two and three dimensions. Numerically obtained profiles at the end of the channel are used for specifying the inflow boundary condition for gas flow. For hydrogen injection, mass flow rate inflow condition is set. The outflow boundary condition is non-reflective and depends on the local Mach number.

The computational grid for 2D calculations consists of 88000 cells. The minimum cell spacing at wall is $5 \cdot 10^{-6} \mathrm{~m}$. In the $3 \mathrm{D}$ case, the number of grid cells is $8.8 \mathrm{M}$. On the sidewalls the cell spacing is also $5 \cdot 10^{-6} \mathrm{~m}$.

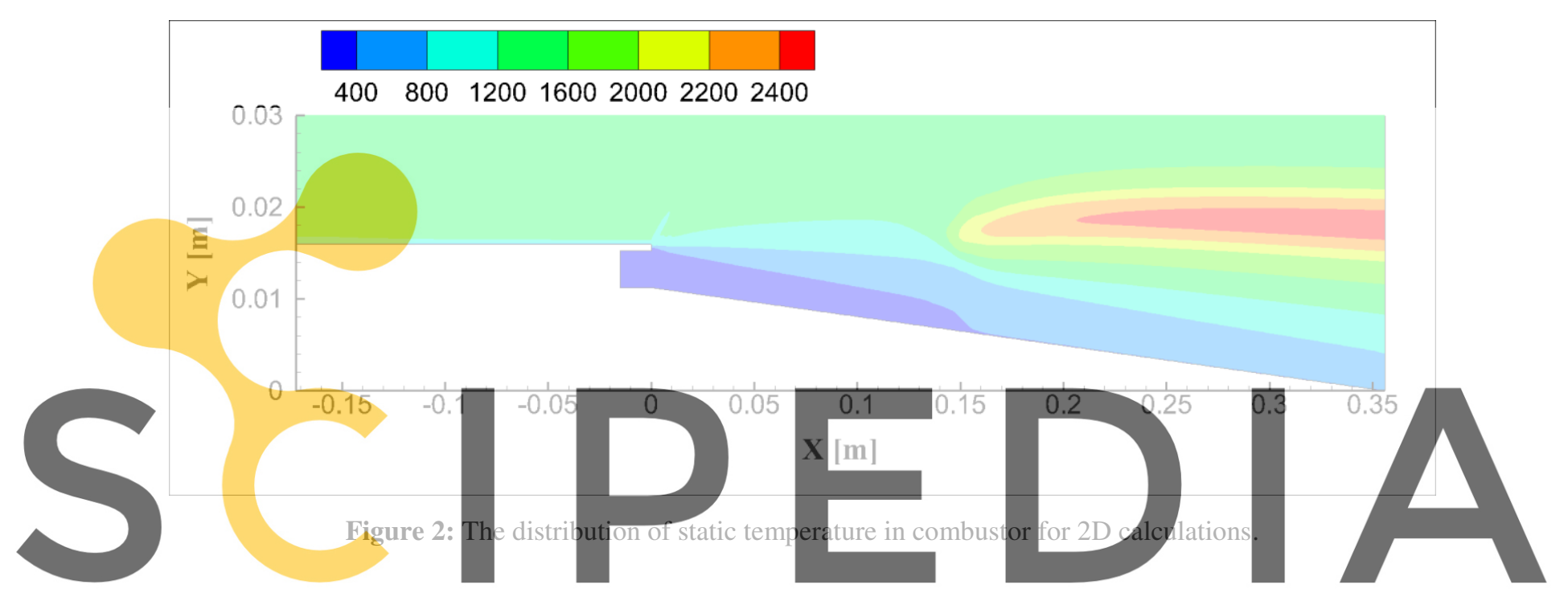

Register for free at https//www.scipedia.com to download the version without the watermark

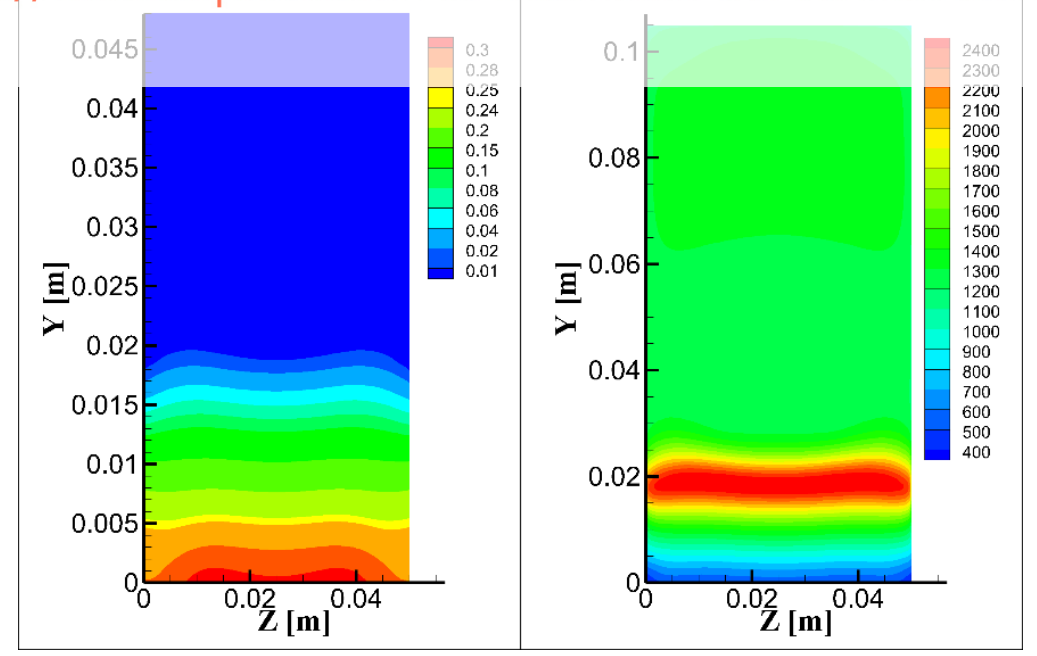

Figure 3: Distributions at the combustor exit: hydrogen mass fraction (left) and static temperature [K] (right). 
We start with the 2D case when the transverse direction is neglected. Fig. 2 shows the distribution of static temperature. The position and geometry of the flame zone can be clear observed here.

Fig. 3 shows numerical results of 3D calculations where the distributions of hydrogen mass fraction and static temperature in the cross-section (YZ-plane) nearby the combustor exit are shown.

The structure of mixing (reaction) zone near the combustor exit is clear seen in this figure. Presence of considerable boundary layers on side walls provides better mixing in this area that results in zone thickness reduction near the walls. The flame zone has a complicated shape; it narrows to the walls due to the heat drain into the wall, and gets thicker in boundary layers zone because of better mixing, and then again reduces in the flow core as the velocity increases and mixing deteriorates.

Afterwards, profiles of total temperature at the combustor exit cross-section and also profiles of components mole fractions are given in Fig. 4 and compared with experimental [1, 2] and computational $[10,18]$ data. The total temperature is related with the static temperature with the following expressions:

$$
\frac{T_{t o t}}{T}=\left(1+\sqrt{\operatorname{Pr}} \frac{\gamma-1}{2} M^{2}\right)
$$

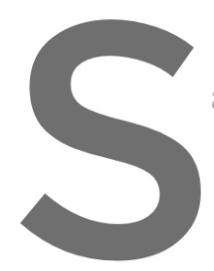

where $\gamma$ is the ratio of specific heats, $M$ is the local Mach number.

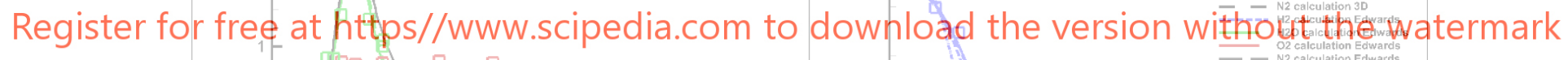
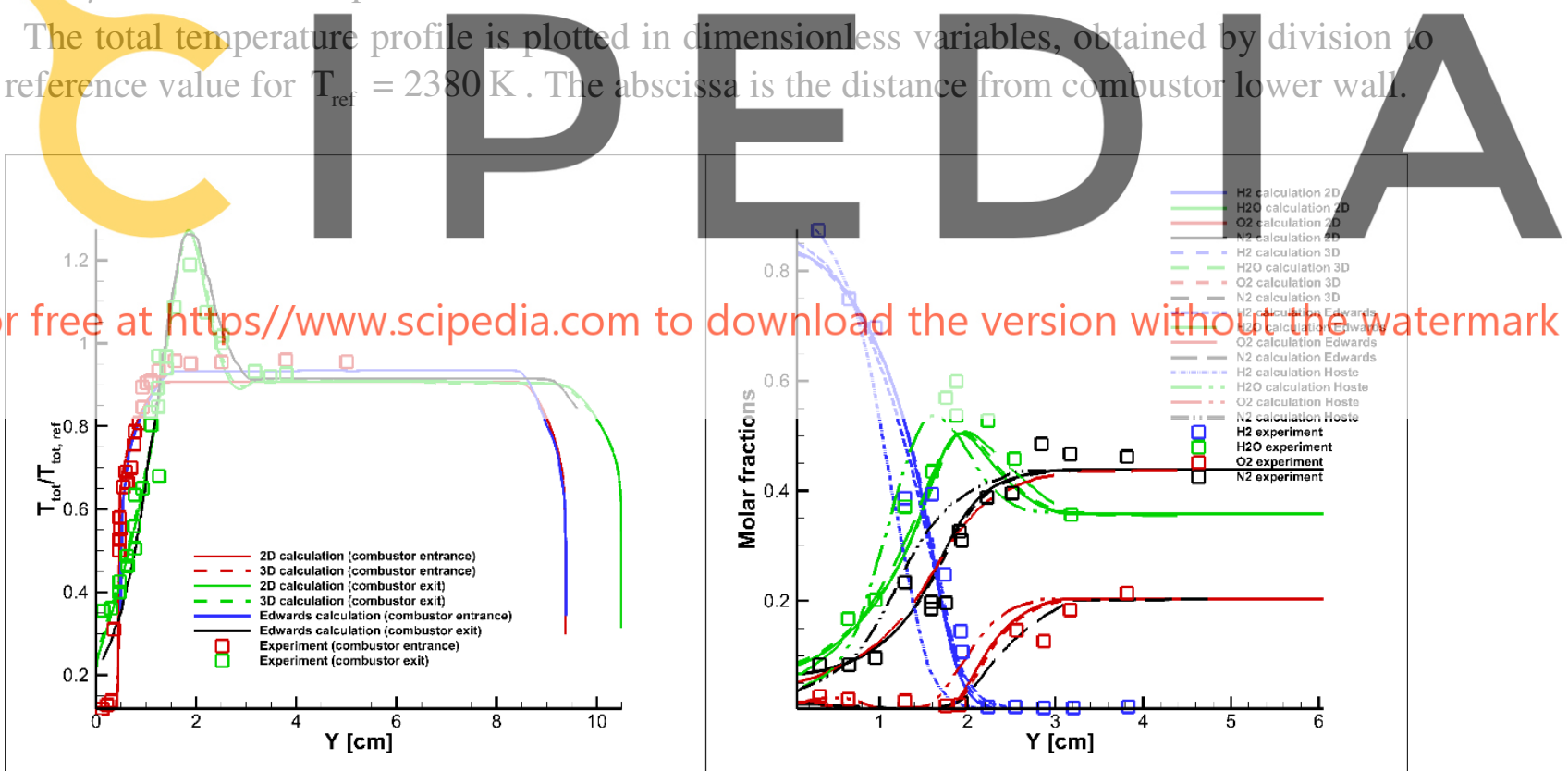

Figure 4: Total temperature and species mole fractions profiles at combustor exit.

The total temperature profiles show very good correlation with corresponding profiles from [10] at combustor exit. The only difference for combustion case is the presence of total temperature small decrease over the flame zone in present calculations.

In Fig. 4, the numerical results are in a good agreement with experimental [2] and numerical [10] data. The results given in [18] have worse correlation with the experiment 
despite of the fact that a more complicated model with variable Prandtl and Schmidt numbers is used in this work.

\section{CONCLUSION}

Numerical modelling of hydrogen injection into the supersonic vitiated air stream with subsequent mixing and combustion in the Burrows-Kurkov chamber has been carried out. The mathematical model used is based on the SA-RANS turbulence supplemented with a diffusion model and coupled with a detailed chemical kinetics mechanism. The system of discrete equations of the hybrid implicit-explicit time marching scheme is effectively solved with the LU-SGS approximate factorization method. Calculations have been performed on grids with precise resolution of boundary layers without using any "wall functions". Total temperature and molar fractions profiles have been compared with experimental and reference numerical data; a good agreement between all the data was observed. This assures that the developed model can be used for predictive modelling of complicated systems involving mixing and combustion in close vicinity of walls.

\section{ACKNOWLEDGEMENTS}

This work was performed within the state assignment of the Scientific Research Institute for System Analysis of the Russian Academy of Sciences (basic scientific research GP 47), subject no. 0580-2021-0005. The work of the first author was supported by RFBR, grant "Aspirant" project number Supercomputer Center RAS clusters.

REFERENCES
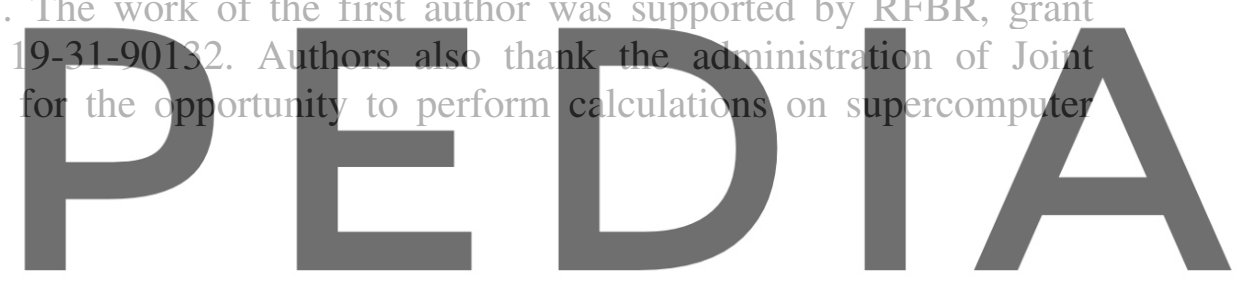

1. Burrows, M.C., and A.P. Kurkov. 1971. Supersonic Combustion of Hydrogen in a

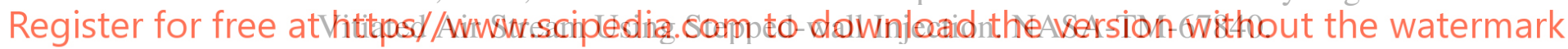

2. Burrows, M.C., and A.P. Kurkov. 1973. Analytical and Experimental Study of Supersonic Combustion of Hydrogen in a Vitiated Airstream. NASA-TM-X-2828.

3. Evans, J.S., Schexnayder, C.J., and H. Beach. 1978. Application of a twodimensional parabolic computer program to prediction of turbulent reacting flows. NASA Technical Paper 1169, 56 p.

4. Evans, J.S., and C.J. Schexnayder. 1980. Influence of Chemical Kinetics and Unmixedness on Burning in Supersonic Hydrogen Flames. AIAA Journal. 1980. Vol. 18. № 2. Pp 188-193.

5. Ebrahimi, H. B. 1994. CFD Validation and Evaluation for Combustor and Nozzle Flow, Part II. AIAA-1994-0025.

6. Engblom, W., Frate, F., and C. Nelson. 2005. Progress in Validation of Wind-US for Ramjet/Scramjet Combustion. AIAA-2005-1000.

7. Gao, Z., Ch. Jiang and Ch.-H. Lee. 2016. On the laminar finite rate model and flamelet model for supersonic turbulent combustion flows. International journal of hydrogen energy, 41. Pp. 13238-13253.

8. Keistler, P.G., Gaffney, R.L., Xiao, X., and H.A. Hassan. 2005. Turbulence Modeling for Scramjet Applications. AIAA-2005-382. 
9. Vyasaprasath, K., Oh, S., Kim, K.-S., and J.-Y. Choi. 2015. Numerical Studies of Supersonic Planar Mixing and Turbulent Combustion using a Detached Eddy Simulation (DES) Model. Int. J. Aeronautical and Space science. 16. Pp. 560-570.

10. Edwards, J.R., J.A. Boles, and R.A. Baurle. 2010. LES / RANS simulation of a supersonic reacting wall jet. AIAA Paper 2010-0370, LF99-10036.

11. Solomatin, R.S., I.V. Semenov, and I.S. Menshov. 2018. Towards calculating turbulent flows with the Spalart-Allmaras model using the LU-SGS-GMRES algorithm. Keldysh Institute for Applied Mathematics preprints № 119. 30 p.

12. Spalart P.R. and S.R. Allmaras. 1992. A one-equation turbulence model for aerodynamic flows. AIAA Paper 1992-0439. 22 p.

13. A.Keromnes et al. 2013. An experimental and detailed chemical kinetic modeling study of hydrogen and syngas mixture oxidation at elevated pressures. Combustion and Flame. - № 160. Pp. 995-1011.

14. J.Troe. 2011. The themal dissociation/recombination reaction of hydrogen peroxide. Analysis and representation of the temperature and pressure dependence over wide ranges. Combustion and Flame. - № 158. Pp. 594-601.

15. Men'shov I.S. and Y. Nakamura. 2004. Unconditionally Stable Scheme for Unsteady Compressible Flows. AIAA Journal. vol. 42. issue 3. Pp. 551-559.

16. Semenov, I.V. and I. Akhmedyanov, Development of parallel LU-SGS algorithm for solution of multidimensional gas dynamics problems. In: Proc. of 4th Siberian
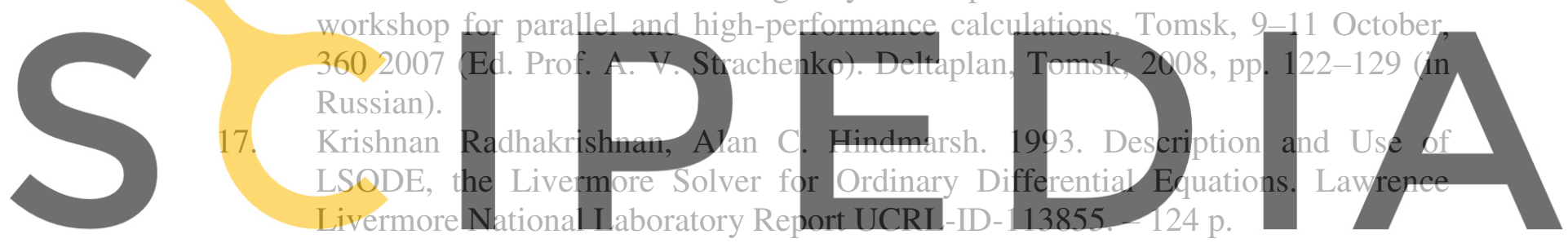

18. Hoste J.J.O.E. et al. 2020. Study of supersonic reaction wall jet with a variable

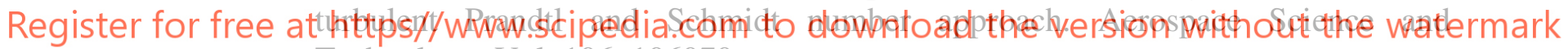
Technology, Vol. 106, 106070. 